\title{
Effects of Tai Chi on exercise capacity and health-related quality of life in patients with chronic obstructive pulmonary disease: a systematic review and meta-analysis
}

REVIEW

\author{
This article was published in the following Dove Press journal: \\ International Journal of COPD \\ 7 November 2014 \\ Number of times this article has been viewed
}

\author{
Weibing Wu' \\ Xiaodan $\mathrm{Liu}^{2}$ \\ Longbing Wang' \\ Zhenwei Wang ${ }^{3}$ \\ Jun $\mathrm{Hu}^{2}$ \\ Juntao $\mathrm{Yan}^{4}$ \\ 'Department of Sports Medicine, \\ Shanghai University of Sport,

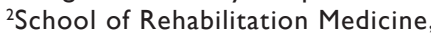 \\ Shanghai University of Traditional \\ Chinese Medicine, ${ }^{3}$ Department of \\ Respiratory Medicine, ${ }^{4}$ Department \\ of Rehabilitation Medicine, Yueyang \\ Hospital of Integrated Traditional \\ Chinese and Western Medicine, \\ Shanghai, People's Republic of China
}

Correspondence: Weibing Wu Department of Sports Medicine, Shanghai University of Sport, Heng Ren Road No I88, Yang Pu District, Shanghai 200438, People's Republic of China

Tel +86 2I 5 I 253248

Fax +86 2I 51253380

Emailwwb75@I26.com
Background: Tai Chi is a traditional Chinese mind-body exercise that has been widely practiced in the People's Republic of China for many centuries. This exercise has also been applied as a training modality in pulmonary rehabilitation programs for stable chronic obstructive pulmonary disease (COPD). This systematic review and meta-analysis aimed to assess the effects of Tai Chi on exercise capacity and health-related quality of life (HRQoL) in COPD patients.

Methods: Electronic databases (PubMed, Embase, Web of Science, The Cochrane Library, Cumulative Index to Nursing and Allied Health Literature, ClinicalTrials.gov, China National Knowledge Infrastructure, and China Biology Medicine disc) were searched. Entries published from January 1980 to March 2014 were included in the search. Eligible studies included those that involved randomized controlled trials and those that lasted for at least 12 weeks. The primary outcome measures were six-minute walking distance (6MWD), St George's Respiratory Questionnaire (SGRQ), and Chronic Respiratory Disease Questionnaire (CRQ). Effect estimates were pooled with random-effects meta-analysis.

Results: Eleven articles involving 824 patients met the inclusion criteria. All included articles compared COPD patients in a Tai Chi group versus COPD patients in nonexercise and/or physical exercise groups. The meta-analysis showed that compared with the nonexercise group, the COPD patients practicing Tai Chi demonstrated significantly enhanced 6MWD (mean difference 35.99 , 95\% confidence interval [CI] 15.63-56.35, $P=0.0005$ ), decreased SGRQ total score (mean difference $-10.02,95 \% \mathrm{CI}-17.59,-2.45, P=0.009$ ), and increased CRQ total score (mean difference $0.95,95 \%$ CI $0.22-1.67, P=0.01$ ). Compared with the physical exercise group, the Tai Chi group showed significantly reduced SGRQ total score (mean difference -3.52 , $95 \% \mathrm{CI}-6.07,-0.97, P=0.007)$, but no statistical significance was found for 6MWD between the two groups (mean difference 13.65, 95\% CI $-1.06,28.37, P=0.07$ ) in COPD patients.

Conclusion: Preliminary evidence suggests that Tai Chi has beneficial effects on exercise capacity and HRQoL in COPD patients. This exercise can be recommended as an effective alternative training modality in pulmonary rehabilitation programs. Further studies are required to support the preliminary evidence and to observe the long-term effects of Tai Chi.

Keywords: traditional Chinese exercise, chronic obstructive pulmonary disease, six-minute walking distance, quality of life

\section{Introduction}

Chronic obstructive pulmonary disease (COPD) is a lung disease that is characterized by incompletely reversible airflow obstruction, which progressively develops and severely endangers human health. Numerous studies have confirmed that pulmonary dysfunction 
reduces exercise capacity in COPD patients, ${ }^{1-3}$ moreover, the decreased exercise capacity further lowers their quality of life. ${ }^{4}$ The six-minute walking distance (6MWD) is a valid indicator for evaluating exercise capacity of COPD patients. ${ }^{5,6}$ Quality of life in COPD patients is usually assessed by the St George's Respiratory Questionnaire (SGRQ) or Chronic Respiratory Disease Questionnaire (CRQ). ${ }^{7}$ Low SGRQ and high CRQ scores are favorable. In recent years, an increasing number of scholars have realized the significance of pulmonary rehabilitation for COPD patients. Physical exercise is the core content of the COPD pulmonary rehabilitation plan. Gimenez et al reported that a maximally intense anaerobic exercise program significantly improved both skeletal and respiratory muscle strength and endurance in COPD patients. ${ }^{8}$ Subin et al reported that combined upper limb and lower limb training achieved a significant increase in 6MWD and CRQ scores in COPD patients. ${ }^{9}$ Moreover, studies from other scholars suggested that long-term physical exercise alleviated symptoms of dyspnea, ${ }^{10}$ enhanced respiratory muscle strength and endurance, ${ }^{10}$ and improved exercise capacity and quality of life in COPD patients. ${ }^{11,12}$ Therefore, the Global Initiative for Chronic Obstructive Lung Disease (GOLD) clearly noted that pulmonary rehabilitation should be included in conventional therapy for the following groups: patients with intermediate and severe COPD; stable COPD patients; patients who suffer from continuing respiratory symptoms; patients with restricted exercise capacity; and patients who have not achieved satisfactory results after medication. ${ }^{13,14}$

Tai Chi is a traditional Chinese exercise that involves human physical activity, breath expiration and inspiration, and mind regulation. Tai Chi also involves soft movements and coordination of respiration and movement. This exercise is easy and enjoyable to learn. Many studies have confirmed that long-term Tai Chi exercises positively affect physical function, exercise capacity, and psychological state; moreover, long-term practice of this exercise helps in the treatment of chronic diseases. ${ }^{15-17}$ For the past few years, Tai Chi has been widely applied in physical rehabilitation of COPD patients. Tai Chi could reduce symptoms of dyspnea, alleviate the decline of lung function, enhance exercise capacity, and improve life quality for patients, as revealed in previous studies. ${ }^{18-20}$ However, some studies have also shown that Tai Chi failed to effectively improve lung function or exercise capacity in COPD patients. ${ }^{21-23}$

Therefore, to further define the effect of Tai Chi in COPD patients, we undertook a systematic review and meta-analysis of Tai Chi as a rehabilitation intervention in COPD patients. We analyzed and evaluated the effect of
Tai Chi on exercise capacity and health-related quality of life (HRQoL) in these patients.

\section{Methods}

\section{Search strategy and selection criteria}

A systematic review of the published literature on Tai Chi intervention in COPD patients was conducted. Studies were identified for inclusion in this review by searching articles that were published from January 1980 to March 2014 in the following databases: PubMed, Embase, Web of Science, The Cochrane Library, ClinicalTrials.gov, Cumulative Index to Nursing and Allied Health Literature, China National Knowledge Infrastructure, and China Biology Medicine disc. We used the following combined text and Medical Subject Headings (MeSH) terms: "Tai Chi", "COPD”, "chronic obstructive pulmonary disease", "pulmonary disease", "exercise capacity", and "quality of life". Search terms used in PubMed were ("tai ji" [MeSH] OR tai ji OR tai chi OR tai ji quan OR qigong OR traditional Chinese exercise) AND ("lung disease, obstructive" [MeSH] OR "pulmonary disease, chronic obstructive" [MeSH] OR COPD OR chronic obstructive lung disease OR bronchiti*, chronic* OR emphysema*) AND (controlled clinical trial [pt] OR randomized controlled trial [pt] OR randomized [tiab] OR placebo [tiab] OR group [tiab] OR randomly [tiab]). To ensure a thorough search of the literature, we also hand-searched references of key articles that were published in English and in Chinese.

Studies were eligible for inclusion in this review if they satisfied the following criteria: randomized controlled trial; used Tai Chi as the activity of the intervention group, used nonexercising patients as the control group, and used patients engaging in exercise (aerobics, strength training, or breathing exercises) as the comparison group; included COPD patients without restrictions in sex, age, and race, and the ratio of forced expiratory volume in one second $\left(\mathrm{FEV}_{1}\right)$ to forced vital capacity was less than $70 \%$ or $\mathrm{FEV}_{1}$ was less than $80 \%$ of predicted values according to the GOLD criteria; the Tai Chi intervention lasted for more than 12 weeks; and outcome measures included 6MWD, SGRQ, or CRQ. Studies that comprised patients who were not recently diagnosed with COPD or enrolled patients with acute exacerbation were excluded.

\section{Data extraction and quality assessment}

Data were independently extracted by two researchers (WW, XL). An agreement value of $91 \%$ in the studies was selected by these two researchers for detailed analysis, and 
disagreements were resolved by a third researcher (LW). For each eligible study, we extracted and recorded the first author's name, year of publication, study design, intervention and control group information, sample size, duration of intervention, and outcomes, including 6MWD, SGRQ, and CRQ results. We contacted the corresponding authors for additional information if necessary.

The quality of each study was independently assessed by two researchers (WW and XL) and was scored according to the corresponding criteria. The methodological quality of each study was evaluated using the Physiotherapy Evidence Database (PEDro) scale. ${ }^{24}$ A score of $\leq 5 / 10$ indicated low quality, whereas a score of $\geq 6 / 10$ indicated high quality. The risk of bias was assessed using the quality criteria of the Cochrane Handbook for Systematic Reviews of Interventions. ${ }^{25}$ All studies were reviewed and assigned with a value of high, low, or unclear in accordance with the recommended criteria of the Cochrane Handbook.

\section{Statistical analysis}

All statistical analyses were performed using the Cochrane Collaboration's Review Manager Software (RevMan version 5.2.0). All collected data were continuous, and the mean difference was calculated using the weighted mean difference in this study. All measures were estimated from each study with associated $95 \%$ confidence intervals (CIs) and pooled studies using a random-effects model to provide estimates of the efficacy of Tai Chi. Eligible studies were included and analyzed using the mean and standard deviations of the change from baseline to endpoint of each intervention period. Heterogeneity across studies was assessed using the $I^{2}$ statistic. Studies with $I^{2}<25 \%$ have low heterogeneity, $I^{2}$ of $25 \%-75 \%$ indicates medium heterogeneity, and $I^{2}>75 \%$ implies high heterogeneity. ${ }^{26}$ If $I^{2}$ was $>50 \%$, sensitivity analyses were conducted to explore the function of the total effect on the outcomes by excluding studies with low methodological quality. A $P$-value less than 0.05 for all outcome measures was considered to be statistically significant.

\section{Results}

\section{Search results}

A total of 98 potentially relevant articles were identified by a literature search and additional hand-searching (Figure 1). Of these articles, 60 duplicates were removed, and 17 were excluded based on title and abstract. Thus, 21 studies were left for further assessment. Finally, eleven articles ${ }^{18,20,21,23,27-33}$ were selected for meta-analysis.

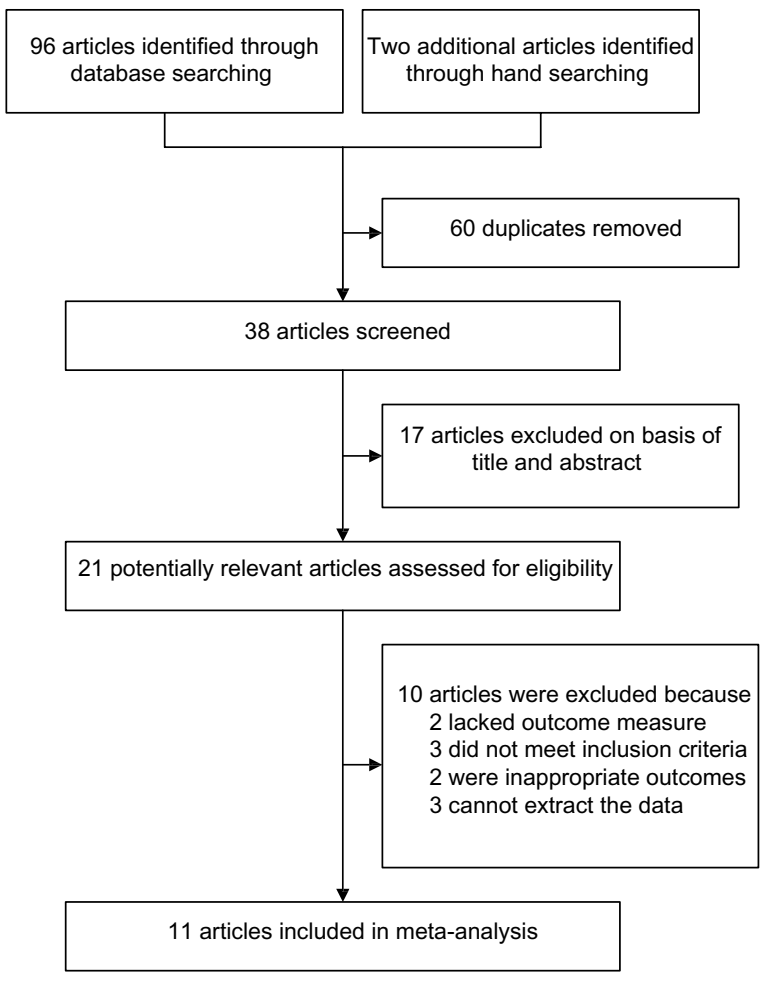

Figure I Flow diagram of article selection process.

\section{Study characteristics}

Table 1 shows the detailed study characteristics of the eleven eligible articles, ${ }^{18,20,21,23,27-33}$ which were retrieved from nine studies. Two articles written by Chan et $\mathrm{al}^{20,27}$ originated from one study, and two articles that were written by $\mathrm{Du}$ et $\mathrm{al}^{30,31}$ also came from a single study. Six studies $^{18,21,23,27-29}$ were published in English, and five ${ }^{22,30-33}$ were published in Chinese. The sample sizes ranged from 10 to 206 (with total of 824 ). Seven studies ${ }^{18,20,23,27,30,31,33}$ reported that the Tai Chi intervention lasted for 12 weeks, and four studies ${ }^{22,28,29,32}$ reported that the treatment lasted for 24 weeks. The frequencies varied from two to five sessions per week, and exercise time lasted 30-60 minutes per session. Seven studies ${ }^{20,22,23,28-30,33}$ measured 6MWD to assess exercise capacity. HRQoL was evaluated by SGRQ in five studies ${ }^{22,27,29,31,32}$ and by CRQ in two studies. ${ }^{18,23}$ Nine studies $^{18,20,22,23,27,28,30,31,33}$ involved both Tai Chi and nonexercise groups, and seven studies ${ }^{20,22,27,29-32}$ involved Tai Chi and physical exercise groups. The control (nonexercise) groups received health education, and comparison of the physical exercise groups was based on aerobics, strength training, or breathing exercises; moreover, these physical exercise groups in most of the included studies performed breathing exercises and aerobics were often based on selfpaced walking activity. 
Table I Characteristics of included studies

\begin{tabular}{|c|c|c|c|c|c|c|c|}
\hline Study & $\begin{array}{l}\text { Study } \\
\text { design }\end{array}$ & $\begin{array}{l}\text { Sample } \\
\text { size }\end{array}$ & $\begin{array}{l}\text { Mean (SD) } \\
\text { age, years }\end{array}$ & $\begin{array}{l}\text { Percent } \\
\text { men }\end{array}$ & Disease severity & Intervention protocol & Outcomes \\
\hline $\begin{array}{l}\text { Chan } \\
\text { et } \mathrm{al}^{27}\end{array}$ & $\begin{array}{l}\text { RCT } \\
\text { (single- } \\
\text { blind) }\end{array}$ & $\begin{array}{l}\text { TC: } 70 \\
\text { PE: } 69 \\
\text { NE: } 67\end{array}$ & $\begin{array}{l}\text { TC: } 71.7(8.2) \\
\text { PE: } 73.6(7.5) \\
\text { NE: } 73.6(7.4)\end{array}$ & $91.0 \%$ & $\begin{array}{l}16 \% \text { mild stage, } \\
42 \% \text { moderate stage, } \\
43 \% \text { severe stage; } \\
\text { mean } \mathrm{FEV}, 0.9 \pm 0.4\end{array}$ & $\begin{array}{l}\text { TC: I3-form TC, } 12 \text { weeks, } \\
\text { twice per week, } 60 \text { minutes per time } \\
\text { PE: PLB, DB, self-paced walking, } \\
\text { I } 2 \text { weeks, I hour per day } \\
\text { NE: routine activities }\end{array}$ & $\begin{array}{l}\text { HRQoL } \\
\text { (SGRQ) }\end{array}$ \\
\hline $\begin{array}{l}\text { Yeh } \\
\text { et } \mathrm{al}^{23}\end{array}$ & $\begin{array}{l}\text { RCT } \\
\text { (single- } \\
\text { blind) }\end{array}$ & $\begin{array}{l}\text { TC: } 5 \\
\text { NE: } 5\end{array}$ & $\begin{array}{l}\text { TC: } 65(6) \\
\text { NE: } 66(6)\end{array}$ & $60.0 \%$ & $\begin{array}{l}\text { Total mean } \mathrm{FEV}_{1} \% \\
\text { predicted } 50 \% \pm 7 \%\end{array}$ & $\begin{array}{l}\text { TC: Yang-style TC, } 12 \text { weeks, } \\
\text { twice per week, } 60 \text { minutes per time } \\
\text { NE: usual care }\end{array}$ & $\begin{array}{l}\text { 6MWD, } \\
\text { HRQoL } \\
\text { (CRQ) }\end{array}$ \\
\hline $\begin{array}{l}\text { Chan } \\
\text { et } \mathrm{al}^{20}\end{array}$ & $\begin{array}{l}\text { RCT } \\
\text { (single- } \\
\text { blind) }\end{array}$ & $\begin{array}{l}\text { TC: } 70 \\
\text { PE: } 69 \\
\text { NE: } 67\end{array}$ & $\begin{array}{l}\text { TC: } 71.7(8.2) \\
\text { PE: } 73.6(7.5) \\
\text { NE: } 73.6(7.4)\end{array}$ & $91.0 \%$ & $\begin{array}{l}16 \% \text { mild stage, } \\
42 \% \text { moderate stage, } \\
43 \% \text { severe stage; } \\
\text { mean } \mathrm{FEV}_{1} 0.9 \pm 0.4\end{array}$ & $\begin{array}{l}\text { TC: I3-form TC, } 12 \text { weeks, } \\
\text { twice per week, } 60 \text { minutes per time } \\
\text { PE: PLB, DB, self-paced walking, } \\
\text { I } 2 \text { weeks, I hour per day } \\
\text { NE: routine activities }\end{array}$ & 6MWD \\
\hline $\begin{array}{l}\text { Zhang } \\
\text { et } \mathrm{al}^{22}\end{array}$ & RCT & $\begin{array}{l}\text { TC: } 30 \\
\text { PE: } 30 \\
\text { NE: } 30\end{array}$ & $\begin{array}{l}\text { TC: } 63.1(7.3) \\
\text { PE: } 62.3(6.9) \\
\text { NE: } 62.0(7.3)\end{array}$ & $56.7 \%$ & $\begin{array}{l}\text { Total mean } \mathrm{FEV}, \% \\
\text { predicted } 52.0 \% \pm 8.2 \%\end{array}$ & $\begin{array}{l}\text { TC: } 24 \text {-form TC, PLB, RMT, } 24 \text { weeks, } \\
\text { once per day, } 30-60 \text { minutes per time } \\
\text { PE: PLB, RMT, } 24 \text { weeks, 3-4 times per day, } \\
\text { I5-30 minutes per time } \\
\text { NE: usual medical care }\end{array}$ & $\begin{array}{l}\text { 6MWD, } \\
\text { HRQoL } \\
\text { (SGRQ), }\end{array}$ \\
\hline $\begin{array}{l}\mathrm{Li} \\
\text { et } \mathrm{al}^{32}\end{array}$ & RCT & $\begin{array}{l}\text { TC: } 35 \\
\text { PE: } 35\end{array}$ & $\begin{array}{l}\text { TC: } 72.0(2.5) \\
\text { PE: } 73.2(3.0)\end{array}$ & $78.6 \%$ & $\begin{array}{l}60 \% \text { moderate stage, } \\
40 \% \text { severe stage }\end{array}$ & $\begin{array}{l}\text { TC: } 24 \text {-form TC, BE, } 24 \text { weeks, } \\
\text { once per day, } 60 \text { minutes per time } \\
\text { PE: BE }\end{array}$ & $\begin{array}{l}\text { HRQoL } \\
\text { (SGRQ) }\end{array}$ \\
\hline $\begin{array}{l}\mathrm{Gu} \\
\text { et } \mathrm{al}^{33}\end{array}$ & RCT & $\begin{array}{l}\text { TC: } 33 \\
\text { NE: } 30\end{array}$ & $\begin{array}{l}\text { TC: } 67(8) \\
\text { NE: } 69 \text { (9) }\end{array}$ & $85.7 \%$ & $\begin{array}{l}\text { Total mean } \mathrm{FEV}, \% \\
\text { predicted } 50.0 \% \pm 19.4 \%\end{array}$ & $\begin{array}{l}\text { TC: } 24 \text {-form TC, } 12 \text { weeks, } 3 \text { times } \\
\text { per week, } 60 \text { minutes per time } \\
\text { NE: health-related knowledge education }\end{array}$ & 6MWD \\
\hline $\begin{array}{l}\text { Leung } \\
\text { et } \mathrm{al}^{18}\end{array}$ & $\begin{array}{l}\text { RCT } \\
\text { (single- } \\
\text { blind) }\end{array}$ & $\begin{array}{l}\text { TC: } 22 \\
\text { NE: } 20\end{array}$ & $\begin{array}{l}\text { TC: } 73(8) \\
\text { NE: } 75(8)\end{array}$ & $64.3 \%$ & $\begin{array}{l}\text { Total mean } \mathrm{FEV}, \% \\
\text { predicted } 59 \% \pm 15 \%\end{array}$ & $\begin{array}{l}\text { TC: Sun-style TC, } 12 \text { weeks, } \\
\text { twice per week, } 60 \text { minutes per time } \\
\text { NE: usual medical care }\end{array}$ & $\begin{array}{l}\text { HRQoL } \\
\text { (CRQ) }\end{array}$ \\
\hline $\begin{array}{l}\text { Niu } \\
\text { et } \mathrm{al}^{28}\end{array}$ & $\begin{array}{l}\text { RCT } \\
\text { (single- } \\
\text { blind) }\end{array}$ & $\begin{array}{l}\text { TC: } 19 \\
\text { NE: } 20\end{array}$ & $\begin{array}{l}\text { TC: } 6 I .3(8.0) \\
\text { NE: } 59.7(2.8)\end{array}$ & $92.5 \%$ & $\begin{array}{l}\text { Total mean } \mathrm{FEV}, \% \\
\text { predicted } 42.8 \% \pm 5.3 \%\end{array}$ & $\begin{array}{l}\text { TC: TC, } 24 \text { weeks, once per day, } \\
50 \text { minutes per time } \\
\text { NE: usual medical care }\end{array}$ & 6MWD \\
\hline $\begin{array}{l}\mathrm{Ng} \\
\text { et } \mathrm{a}^{29}\end{array}$ & $\begin{array}{l}\text { RCT } \\
\text { (single- } \\
\text { blind) }\end{array}$ & $\begin{array}{l}\text { TC: } 94 \\
\text { PE: } 98\end{array}$ & $\begin{array}{l}\text { TC: } 74.1 \text { (6.8) } \\
\text { PE: } 74.2(6.5)\end{array}$ & $91.2 \%$ & $\begin{array}{l}20.5 \% \text { mild stage, } \\
40.6 \% \text { moderate stage, } \\
30.2 \% \text { severe stage; } \\
\text { total mean } \mathrm{FEV} \% \\
\text { predicted } 59.8 \% \pm 23.2 \%\end{array}$ & $\begin{array}{l}\text { TC: Sun-style TC, PRP, } 24 \text { weeks, } \\
5-7 \text { times per week, } 80 \text { minutes per time } \\
\text { PE: PRP (aerobics, BE, strength training), } \\
24 \text { weeks, } 5-7 \text { times per week, } \\
80 \text { minutes per time }\end{array}$ & $\begin{array}{l}\text { 6MWD } \\
\text { HRQoL } \\
\text { (SGRQ) }\end{array}$ \\
\hline $\begin{array}{l}\mathrm{Du} \\
\text { et } \mathrm{al}^{30}\end{array}$ & RCT & $\begin{array}{l}\text { TC: } 36 \\
\text { PE: } 38 \\
\text { NE: } 38\end{array}$ & $\begin{array}{l}\text { TC: } 65.2(8.3) \\
\text { PE: } 62.4(6.2) \\
\text { NE: } 64.5(6.5)\end{array}$ & $62.5 \%$ & $\begin{array}{l}29.5 \% \text { mild stage, } \\
70.5 \% \text { moderate stage; } \\
\text { total mean } \mathrm{FEV}_{1} \% \\
\text { predicted } 73.54 \% \pm 6.73 \%\end{array}$ & $\begin{array}{l}\text { TC: } 24 \text {-form Yang-style TC, } 12 \text { weeks, } \\
\text { once per day, } 60 \text { minutes per time } \\
\text { PE: PLB, DB, walking exercise, } 12 \text { weeks, } \\
\text { I hour PLB and DB, } 30 \text { minutes } \\
\text { walking per day } \\
\text { NE: routine activities }\end{array}$ & 6MWD \\
\hline $\begin{array}{l}\mathrm{Du} \\
\text { et } \mathrm{al}^{31}\end{array}$ & $\mathrm{RCT}$ & $\begin{array}{l}\text { TC: } 36 \\
\text { PE: } 38 \\
\text { NE: } 38\end{array}$ & $\begin{array}{l}\text { TC: } 65.2(8.3) \\
\text { PE: } 62.4(6.2) \\
\text { NE: } 64.5(6.5)\end{array}$ & $62.5 \%$ & $\begin{array}{l}29.5 \% \text { mild stage, } \\
70.5 \% \text { moderate stage; } \\
\text { total mean } \mathrm{FEV}, \% \\
\text { predicted } 73.54 \% \pm 6.73 \%\end{array}$ & $\begin{array}{l}\text { TC: } 24 \text {-form Yang-style TC, } 12 \text { weeks, } \\
\text { once per day, } 60 \text { minutes per time } \\
\text { PE: PLB, DB, walking exercise, } 12 \text { weeks, } \\
\text { I hour PLB and DB, } 30 \text { minutes } \\
\text { walking per day } \\
\text { NE: routine activities }\end{array}$ & $\begin{array}{l}\text { HRQoL } \\
\text { (SGRQ) }\end{array}$ \\
\hline
\end{tabular}

Abbreviations: RCT, randomized controlled trial; TC, Tai Chi group; NE, nonexercise group; PE, physical exercise group; SD, standard deviation; FEV , forced expiratory volume in one second; PRP, pulmonary rehabilitation program; PLB, pursed-lip breathing; RMT, respiratory muscle training; BE, breathing exercise; DB, diaphragmatic breathing; 6MWD, six-minute walking distance; HRQoL, health-related quality of life; SGRQ, St George's Respiratory Questionnaire; CRQ, Chronic Respiratory Disease Questionnaire.

\section{Quality of included studies}

Table 2 shows assessment of the included studies ${ }^{18,20,22,23,28-30,32,33}$ for methodology by PEDro. The average quality score was 5.7 out of a possible 10 (in the range 4-8). All studies reported no significant differences in baseline age, sex, body mass index, and disease course between the Tai Chi group and nonexercise or physical exercise groups. All studies used appropriate randomization strategies and described a randomized method including random number table, random number generator, and drawing lots. Only one ${ }^{18}$ of the nine 


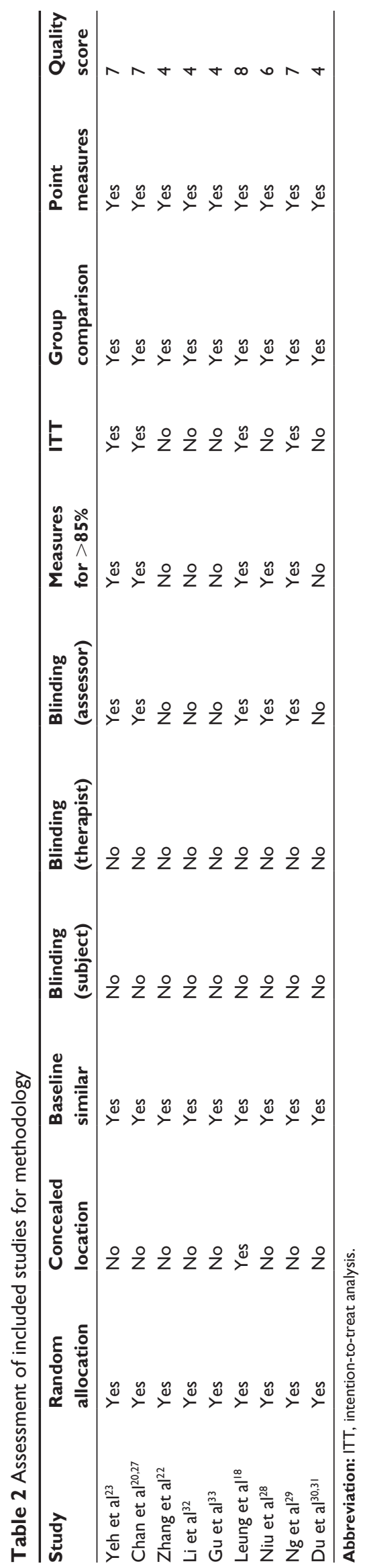

studies had adequate intervention allocation concealment, and five studies ${ }^{18,20,28,29,32}$ masked the intervention from the outcome assessor. Six ${ }^{18,20,28,29,32,33}$ of the nine studies mentioned withdrawals, and four ${ }^{18,20,23,29}$ performed an intentionto-treat analysis. Table 3 shows an assessment of the included studies $^{18,20,22,23,28-30,32,33}$ for risk of bias using the recommended criteria of the Cochrane Handbook. ${ }^{25}$

\section{Meta-analyses of outcome measures for 6MWD}

In a pooled analysis of the six studies $(\mathrm{n}=383),{ }^{20,22,23,28,30,33}$ the 6MWD was significantly enhanced in the Tai Chi group (mean difference $35.99 \mathrm{~m}, 95 \%$ CI 15.63-56.35, $P=0.0005$ ) compared with the nonexercise group (Figure 2A). In a pooled analysis of the four studies $(n=465)^{20,22,29,30}$ of Tai Chi versus physical exercise groups, no significant difference was found in 6MWD (mean difference $13.65 \mathrm{~m}, 95 \% \mathrm{CI}-1.06$, 28.37, $P=0.07$ ) using a random-effects model (Figure $2 \mathrm{~B}$ ).

A sensitivity analysis that excluded studies with low quality for 6MWD according to the criteria of PEDro was also conducted. Table 4 shows the results of sensitivity analyses in the Tai Chi group and nonexercise or physical exercise groups. Sensitivity analyses that excluded the three low quality studies of Zhang et al, ${ }^{22} \mathrm{Du}$ et al, ${ }^{30}$ and $\mathrm{Gu}$ et $\mathrm{al}^{33}$ for 6MWD failed to alter the pooled results in the Tai Chi and nonexercise groups. However, a significant change in 6MWD was found between the Tai Chi and physical exercise groups when the two low quality studies of Zhang et a ${ }^{12}$ and Du et a ${ }^{30}$ were removed.

\section{Meta-analyses of outcome measures for HRQoL}

In a pooled analysis of three studies $(n=271), 22,27,31$ the Tai Chi group showed a significantly decreased SGRQ total score (mean difference $-10.02,95 \% \mathrm{CI}-17.59,-2.45, P=0.009$ ) compared with the nonexercise group (Figure $3 \mathrm{~A}$ ). In a pooled analysis of five studies ( $\mathrm{n}=525), 22,27,29,31,32$ the Tai Chi group showed a significantly decreased SGRQ total score (mean difference $-3.52,95 \% \mathrm{CI}-6.07,-0.97, P=0.007$ ) compared with the physical exercise group (Figure $3 \mathrm{~B}$ ). In a pooled analysis of two studies $(\mathrm{n}=52),{ }^{18,23}$ the Tai Chi group significantly increased the CRQ total score (mean difference $0.95,95 \% \mathrm{CI} 0.22-1.67, P=0.01$ ) compared with the nonexercise group (Figure 4).

A meta-analysis of domain scores on the SGRQ was also conducted, which included symptoms, activity, and influence (Table 5). The pooled mean differences from three studies $^{22,27,31}$ were $-9.91(95 \% \mathrm{CI}-14.16,-5.66, P<0.00001)$ for symptom scores, -11.15 (95\% CI $-23.23,0.92, P=0.07)$ 
Table 3 Assessment of included studies for risk of bias

\begin{tabular}{|c|c|c|c|c|c|c|c|}
\hline Study & $\begin{array}{l}\text { Adequate } \\
\text { sequence } \\
\text { generation }\end{array}$ & $\begin{array}{l}\text { Allocation } \\
\text { concealment }\end{array}$ & $\begin{array}{l}\text { Blinding of } \\
\text { participants } \\
\text { and personnel }\end{array}$ & $\begin{array}{l}\text { Blinding of } \\
\text { outcome } \\
\text { assessment }\end{array}$ & $\begin{array}{l}\text { Incomplete } \\
\text { outcome } \\
\text { data }\end{array}$ & $\begin{array}{l}\text { Selective } \\
\text { reporting }\end{array}$ & $\begin{array}{l}\text { Other } \\
\text { bias }\end{array}$ \\
\hline Yeh et $\mathrm{al}^{23}$ & Low & Unclear & Unclear & Low & Low & Low & Unclear \\
\hline Chan et $\mathrm{al}^{20,27}$ & Low & Unclear & Unclear & Low & Low & Low & Unclear \\
\hline Zhang et $\mathrm{al}^{22}$ & Unclear & Unclear & Unclear & Unclear & Low & Low & Unclear \\
\hline Li et $\mathrm{al}^{32}$ & Low & Unclear & Unclear & Unclear & Low & Low & Unclear \\
\hline Gu et $\mathrm{al}^{33}$ & High & Unclear & Unclear & Unclear & Low & Low & Unclear \\
\hline Leung et $\mathrm{al}^{18}$ & Low & Low & Unclear & Low & Low & Low & Unclear \\
\hline Niu et $\mathrm{al}^{28}$ & Low & Unclear & Unclear & Low & Low & Low & Unclear \\
\hline $\mathrm{Ng}$ et $\mathrm{al}^{29}$ & Low & Unclear & Unclear & Low & Low & Low & Unclear \\
\hline Du et $\mathrm{al}^{30,31}$ & Low & Unclear & Unclear & Unclear & Low & Low & Unclear \\
\hline
\end{tabular}

for activity scores, and $-10.11(95 \%$ CI $-19.60,-0.62$, $P=0.04$ ) for influence scores in the Tai Chi group versus the nonexercise group. Compared with the nonexercise group, the Tai Chi group showed significantly decreased SGRQ symptom and influence scores. The pooled mean differences from five studies ${ }^{22,27,29,31,32}$ were -1.45 (95\% CI $-4.12,1.22$, $P=0.29)$ for symptom score, -5.06 (95\% CI -8.75 to -1.56 , $P=0.005)$ for activity score, and $-2.11(95 \% \mathrm{CI}-4.46,0.23$,
$P=0.08)$ for influence score in the Tai Chi group versus the physical exercise group. Compared with the physical exercise group, the Tai Chi group showed a significantly decreased activity score of SGRQ $(P=0.005)$.

\section{Discussion}

We conducted a systematic review and meta-analysis to estimate the effects of Tai Chi on exercise capacity and HRQoL

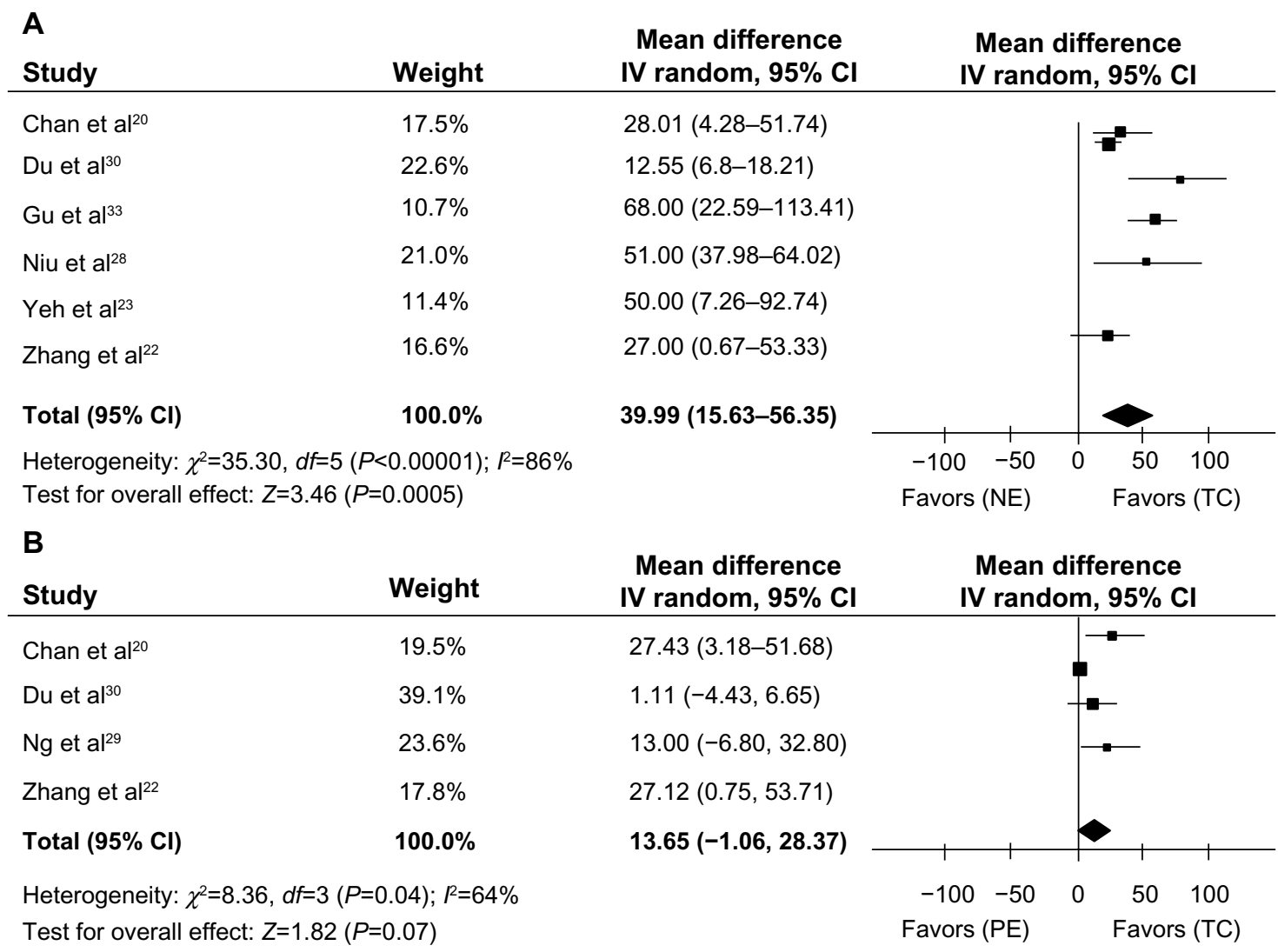

Figure 2 Meta-analysis of the effect of Tai Chi on 6MWD in COPD patients.

Notes: (A) Tai Chi group versus nonexercise group; (B) Tai Chi group versus physical exercise group.

Abbreviations: COPD, chronic obstructive pulmonary disease; TC, Tai Chi group; NE, nonexercise group; PE, physical exercise group; Cl, confidence interval; 6MWD, six-minute walking distance. 
Table 4 Sensitivity analyses of included studies for 6MWD

\begin{tabular}{|c|c|c|c|c|c|c|}
\hline & Studies & $N(n)$ & MD (95\% Cl) & $P$-value & $I^{2}(\%)$ & $\boldsymbol{P}_{\text {heterogeneity }}$ \\
\hline TC versus & All included studies & $6(383)$ & $35.99(15.63,56.35)$ & 0.0005 & $86 \%$ & $<0.0000 \mathrm{I}$ \\
\hline \multirow[t]{3}{*}{ NE } & High quality studies & $3(186)$ & $44.08(28.80,59.37)$ & $<0.00001$ & $29 \%$ & 0.25 \\
\hline & (PEDro $\geq 6 / 10$ ) & & & & & \\
\hline & $\begin{array}{l}\text { Low quality studies } \\
\text { (PEDro } \leq 5 / / 0)\end{array}$ & $3(197)$ & $27.72(2.64,52.8 I)$ & 0.03 & $70 \%$ & 0.04 \\
\hline $\mathrm{TC}$ versus & All included studies & $4(4 \mid I)$ & | $3.87(-1.49,29.23)$ & 0.08 & $63 \%$ & 0.04 \\
\hline \multirow[t]{2}{*}{ PE } & $\begin{array}{l}\text { High quality studies } \\
\text { (PEDro } \geq 6 / 10 \text { ) }\end{array}$ & $2(33 I)$ & $18.77(3.44,34.11)$ & 0.02 & $0 \%$ & 0.37 \\
\hline & $\begin{array}{l}\text { Low quality studies } \\
\text { (PEDro } \leq 5 / / 0)\end{array}$ & $2(134)$ & $10.72(-13.79,35.24)$ & 0.39 & $72 \%$ & 0.06 \\
\hline
\end{tabular}

Abbreviations: TC, Tai Chi group; NE, non exercise group; PE, physical exercise group; PEDro, Physiotherapy Evidence Database; N, number of studies; n, number of patients; MD, mean difference; $\mathrm{Cl}$, confidence interval; 6MWD, six-minute walking distance.

in COPD patients. Decreased exercise capacity is among the main symptoms in COPD patients. Therefore, assessment of exercise capacity helps evaluate motor function, quality of life, and prognosis in these patients. The 6MWD is commonly used in the assessment of the exercise capacity in patients with clinically stable COPD. The 6MWD is also correlated with lung function and can reflect changes in lung function in COPD patients. ${ }^{5,34-36}$ In the analysis of 6MWD in six studies, ${ }^{20,22,23,28,30,33}$ pooled estimates showed a statistically significant increase in the Tai Chi group compared with the nonexercise group. However, the results should be further compared with the minimum clinically important difference (MCID) because not all statistically significant differences were clinically relevant in interpretation of clinical outcome measures. ${ }^{37-40}$ According to the MCID for 6MWD in COPD patients, which was greater than $25 \mathrm{~m},{ }^{41} 35 \mathrm{~m},{ }^{6}$ or $53 \mathrm{~m},{ }^{42}$ the changes in 6MWD in the study by Gu et $\mathrm{al}^{33}$ were greater than the MCID ( $\geq 53 \mathrm{~m}$ ). The changes in 6MWD for the studies by Yeh et $a l,{ }^{23} \mathrm{Niu}$ et $\mathrm{al},{ }^{28}$ and $\mathrm{Gu}$ et $\mathrm{al}^{33}$ were greater than the MCID ( $\geq 35 \mathrm{~m}$ ). The changes in 6MWD for the studies

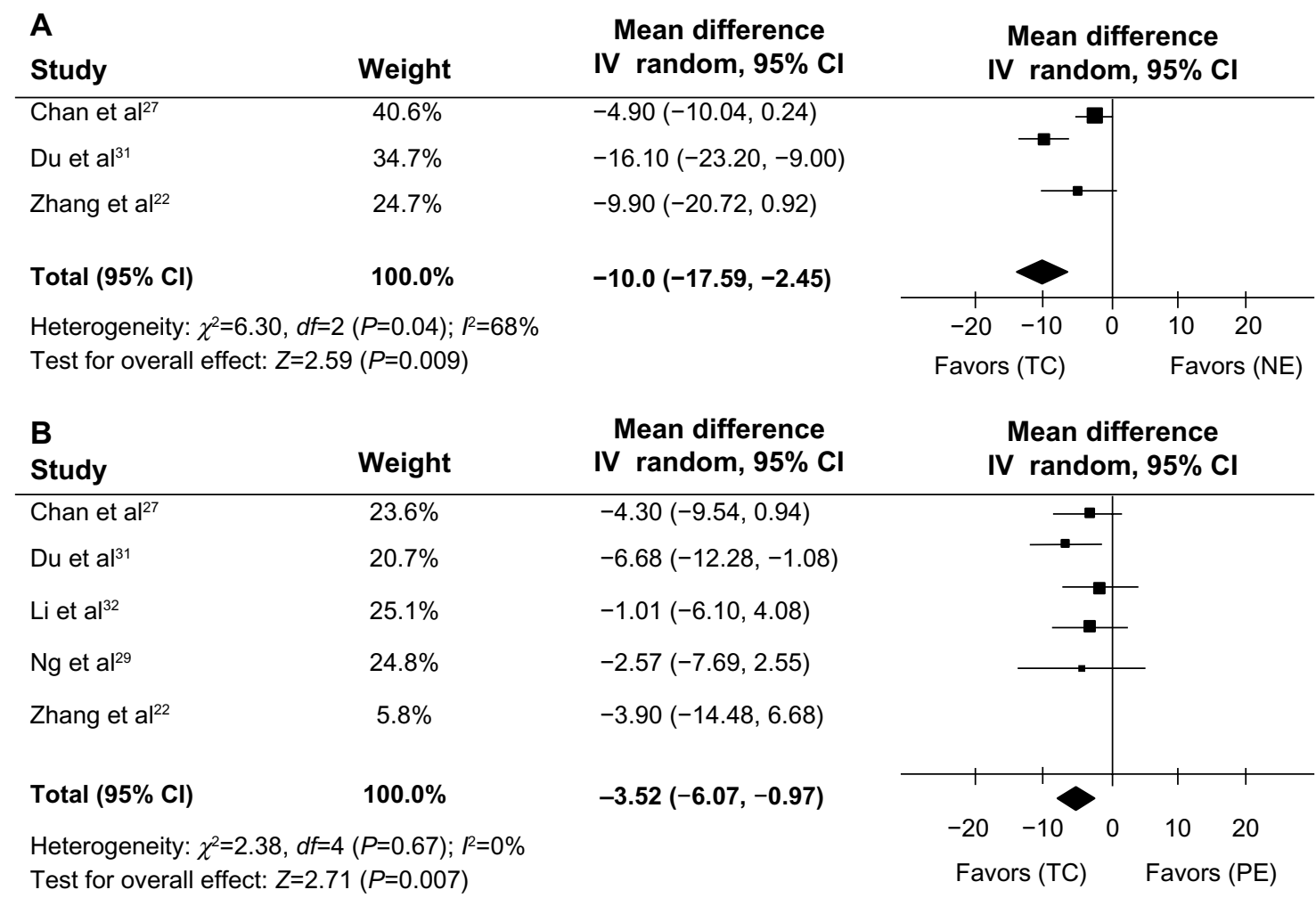

Figure 3 Meta-analysis of the effect of Tai Chi on SGRQ total score in COPD patients.

Notes: (A) Tai Chi group versus nonexercise group. (B) Tai Chi group versus physical exercise group.

Abbreviations: COPD, chronic obstructive pulmonary disease; TC, Tai Chi group; NE, nonexercise group; PE, physical exercise group; Cl, confidence interval; SGRQ, St George's Respiratory Questionnaire. 


\begin{tabular}{|c|c|c|c|c|c|}
\hline Difference study Cl & Weight & $\begin{array}{c}\text { Mean difference } \\
\text { IV random, } 95 \% \mathrm{CI}\end{array}$ & \multicolumn{3}{|c|}{$\begin{array}{c}\text { Mean difference } \\
\text { IV random, } 95 \% \mathrm{CI}\end{array}$} \\
\hline Leung et $\mathrm{al}^{18}$ & $69.0 \%$ & $0.70(0.36-1.04)$ & & \multicolumn{2}{|l|}{$\square$} \\
\hline Yeh et $\mathrm{al}^{23}$ & $31.0 \%$ & $1.50(0.47-2.53)$ & & & \\
\hline Total $(95 \% \mathrm{Cl})$ & $100.0 \%$ & $0.95(0.22-1.67)$ & & & \\
\hline \multirow{2}{*}{\multicolumn{3}{|c|}{ Heterogeneity: $\chi^{2}=2.10, d f=1(P=0.15) ; I^{2}=52 \%$}} & \multirow{2}{*}{$\begin{array}{cc}-4 & -2 \\
\text { Favors (NE) }\end{array}$} & 0 & 4 \\
\hline & & & & Favors & $(\mathrm{TC})$ \\
\hline
\end{tabular}

Figure 4 Meta-analysis of the effect of Tai Chi on CRQ total score in patients with COPD.

Note: Tai Chi group versus no exercise group.

Abbreviations: COPD, chronic obstructive pulmonary disease; TC, Tai Chi group; NE, non exercise group; Cl, confidence interval; CRQ, Chronic Respiratory Disease Questionnaire.

of Chan et al, ${ }^{20}$ Zhang et al, ${ }^{22}$ Yeh et al, ${ }^{23}$ Niu et al, ${ }^{28}$ and $\mathrm{Gu}$ et $\mathrm{al}^{33}$ were greater than the MCID $(\geq 25 \mathrm{~m})$. The mean 6MWD changes in the six studies ${ }^{20,22,23,28,30,33}$ were $35.99 \mathrm{~m}$ and greater than the MCID ( $\geq 35 \mathrm{~m}$ ). However, pooled estimates from four studies ${ }^{20,22,29,31}$ showed no statistically significant difference in 6MWD between the Tai Chi group and the physical exercise group. Therefore, we conducted a quality assessment for the four studies ${ }^{20,22,29,31}$ by methodology, and two studies ${ }^{20,29}$ were determined as being of high quality and the other two ${ }^{22,31}$ as low quality. In pooled analysis of the two high quality studies, Tai Chi significantly increased the $6 \mathrm{MWD}$ compared with physical exercise, but the mean changes in 6MWD were $18.77 \mathrm{~m}$ and were lower than the MCID ( $\geq 25 \mathrm{~m}$ ). Compared with the Tai Chi group, the physical exercise group in the two high quality studies mainly practiced breathing techniques combined with walking exercise; thus, this result for 6MWD differed between the Tai Chi group and the physical exercise group.

HRQoL is an important indicator for assessing the therapeutic effect of Tai Chi in patients with COPD. ${ }^{7,27,31,43,44}$ In the analysis of HRQoL in five studies (three using the $\mathrm{SGRQ}^{22,27,31}$ and two using the $\mathrm{CRQ}^{18,23}$ ), pooled estimates showed a statistically significant decrease in SGRQ total score and an increase in CRQ total score for the Tai Chi group compared with the nonexercise group. Tai Chi significantly improved HRQoL in COPD patients. According to the MCID for the SGRQ total score in COPD patients, which was greater than $4,{ }^{45}$ the mean decrease in SGRQ total score for the three studies ${ }^{22,27,31}$ was 10.02 , which was greater than the MCID ( $\geq 4)$. According to the MCID of the CRQ total score, which was greater than 0.5 in COPD patients, ${ }^{46}$ the mean increase in CRQ total score in the two studies ${ }^{18,23}$ was 0.95 , which was greater than the MCID ( $\geq 0.5)$. Tai Chi produced a statistically and clinically significant improvement in HRQoL in COPD patients. Previous studies clarified that Tai Chi is a moderate-intensity aerobic exercise and emphasizes the overall adjustment in patients with physical, mental, and psychological status, thereby contributing to further improvement in quality of life for patients. ${ }^{27,30,47}$ This meta-analysis shows that patients practicing Tai Chi had improved HRQoL compared with those who did not exercise, but some studies dispute the idea that Tai Chi improves HRQoL when compared with other physical exercise measures. Du et $\mathrm{a}^{31}$ reported that Tai Chi and physical exercise can improve HRQoL in COPD patients and that Tai Chi was better than physical exercise in improving HRQoL.

Table 5 Meta-analyses of domain scores of SGRQ

\begin{tabular}{|c|c|c|c|c|c|c|}
\hline & Outcome & $N(n)$ & MD (95\% Cl) & $P$-value & $I^{2}(\%)$ & $\boldsymbol{P}_{\text {heterogeneity }}$ \\
\hline$\overline{\mathrm{TC} \text { versus }}$ & Symptoms & $3(27 I)$ & $-9.91(-14.16,-5.66)$ & $<0.00001$ & $0 \%$ & 0.76 \\
\hline \multirow[t]{2}{*}{ NE } & Activity & $3(27 I)$ & $-11.15(-23.23,0.92)$ & 0.07 & $82 \%$ & 0.004 \\
\hline & Influence & $3(27 I)$ & $-10.11(-19.60,-0.62)$ & 0.04 & $84 \%$ & 0.02 \\
\hline $\mathrm{TC}$ versus & Symptoms & $5(525)$ & $-1.45(-4.12,1.22)$ & 0.29 & $0 \%$ & 0.96 \\
\hline \multirow[t]{2}{*}{ PE } & Activity & $5(525)$ & $-5.06(-8.75,-1.56)$ & 0.005 & $27 \%$ & 0.24 \\
\hline & Influence & $5(525)$ & $-2.11(-4.46,0.23)$ & 0.08 & $0 \%$ & 0.42 \\
\hline
\end{tabular}

Abbreviations: TC, Tai Chi group; NE, nonexercise group; PE, physical exercise group; N, number of studies; n, number of patients; MD, mean difference; Cl, confidence interval; SGRQ, St George's Respiratory Questionnaire. 
However, Chan et $\mathrm{al}^{27}$ reported no significant difference in HRQoL (SGRQ total score) between patients practicing Tai Chi and those undertaking physical exercise.

This systematic review has several limitations. First, four of the nine included studies were assessed as low quality by PEDro. Thus, low methodological quality might affect the strength of the evidence. Second, data were not pooled from all studies. For example, only two studies contributed to the meta-analysis of the effect of Tai Chi on CRQ total score in COPD patients. Third, the intervention time, frequency, and intensity were not similar between the Tai Chi and physical exercise groups, which was likely to affect the reliability of the pooled results. Finally, given that only studies published in Chinese and English were searched, the included studies could be incomplete. Our results should be interpreted with caution in view of these limitations.

We believe that future studies could benefit from the assessment of the effects of Tai Chi on COPD patients in this meta-analysis. First, studies should give detailed reports on random sequence generation and allocation concealment, thereby ensuring better comparability between Tai Chi and nonexercise or physical exercise groups, as well as reducing selection bias. Second, Tai Chi should be simplified to facilitate elderly COPD patients to master the elements of Tai Chi. Such simplification would benefit the application and popularity of Tai Chi in COPD patients. Third, the intervention and follow-up duration of most of the studies that were reviewed was short, and the long-term intervention effect remains unknown. Future studies should consider long-term intervention using this exercise to observe the long-term curative effect of Tai Chi in COPD patients. Finally, exercise intensity should be strengthened, depending on the physical status in of the COPD patient, although this is a controversial issue in COPD pulmonary rehabilitation.

Butcher et $\mathrm{al}^{48}$ proposed that compared with low-intensity sports training, high-intensity exercise could lead to more pronounced pathological and physiological changes in COPD patients. GOLD also emphasized that in the premise of keeping safe, COPD pulmonary rehabilitation should apply training of high and intermediate intensity. ${ }^{2}$ However, a study showed that high-intensity exercise is not suitable for patients with severe COPD. ${ }^{14}$ In most COPD patients, exercise of intermediate and low intensity is more practical than highintensity exercise. Therefore, the exercise intensity should be changed by adapting the COPD patients' height of body gravity when practicing Tai Chi, to observe the rehabilitation effect of Tai Chi at various intensities.

\section{Conclusion}

In conclusion, the findings of this review suggest that Tai Chi may improve exercise capacity and HRQoL in COPD patients. Tai Chi can be recommended as a safe and effective alternative training modality in pulmonary rehabilitation programs. However, considering the limited quantities and low methodological quality of the included studies, further research with larger sample sizes and longer-term intervention are needed to confirm the effect of Tai Chi on the rehabilitation of COPD patients.

\section{Acknowledgments}

This study was supported by the Key Laboratory of Exercise and Health Sciences, Ministry of Education, Shanghai University of Sport (yk2013001), the research project of the Fitness Qigong Administrative Centre of General Administration of Sport of China (QG2013003), the Chinese medicine research project of the Shanghai Municipal Commission of Health and Family Planning (2014LQ081A), and the budgetary project of Shanghai University of Traditional Chinese Medicine (2012JW16).

\section{Author contributions}

WW, XL, and JH conceived the study. XL, LW, ZW, JH, and JY selected studies and abstracted data. LW and ZW did the statistical analysis. WW wrote the first draft and all authors contributed to the writing of the final report.

\section{Disclosure}

The authors report no conflicts of interest in this work.

\section{References}

1. Chronic Obstructive Pulmonary Disease Committee, Respiratory Society, Chinese Medical Association. [Diagnosis and treatment guidelines of chronic obstructive pulmonary disease]. Chin J Tuberc Respir Dis. 2007;30(1):8-17. Chinese.

2. Lin WC, Yuan SC, Chien JY, Weng SC, Chou MC, Kuo HW. The effects of respiratory training for chronic obstructive pulmonary disease patients: a randomised clinical trial. J Clin Nurs. 2012;21(19-20):2870-2878.

3. Crisafulli E, Venturelli E, Biscione G, et al. Exercise performance after standard rehabilitation in COPD patients with lung hyperinflation. Intern Emerg Med. 2014;9(1):23-31.

4. Zhu Z, Zhang SQ. [Effects of Qigong on respiratory function and quality of life in patients with stable chronic obstructive pulmonary disease]. Jilin Journal of Traditional Chinese Medicine. 2012;32(8):803-804. Chinese.

5. Spruit MA, Polkey MI, Celli B, et al; Evaluation of COPD Longitudinally to Identify Predictive Surrogate Endpoints (ECLIPSE) study investigators. Predicting outcomes from 6-minute walk distance in chronic obstructive pulmonary disease. J Am Med Dir Assoc. 2012;13(3):291-297.

6. Puhan MA, Mador MJ, Held U, Goldstein R, Guyatt GH, Schünemann HJ. Interpretation of treatment changes in 6 minute walk distance in patients with COPD. Eur Respir J. 2008;32(3):637-643. 
7. Puhan MA, Guyatt GH, Goldstein R, et al. Relative responsiveness of the Chronic Respiratory Questionnaire, St Georges Respiratory Questionnaire and four other health-related quality of life instruments for patients with chronic lung disease. Respir Med. 2007;101(2): 308-316.

8. Gimenez M, Servera E, Vergara P, Bach JR, Polu JM. Endurance training in patients with chronic obstructive pulmonary disease: a comparison of high versus moderate intensity. Arch Phys Med Rehabil. 2000;81(1):102-109.

9. Subin, Rao V, Prem V, Sahoo. Effect of upper limb, lower limb and combined training on health-related quality of life in COPD. Lung India. 2010;27(1):4-7.

10. Zwerink M, van der Palen J, van der Valk P, Brusse-Keizer M, Effing T. Relationship between daily physical activity and exercise capacity in patients with COPD. Respir Med. 2013;107(2):242-248.

11. Burtin C, Saey D, Saglam M, et al. Effectiveness of exercise training in patients with COPD: the role of muscle fatigue. Eur Respir J. 2012;40(2):338-344.

12. Probst VS, Kovelis D, Hernandes NA, Camillo CA, Cavalheri V, Pitta F. Effects of 2 exercise training programs on physical activity in daily life in patients with COPD. Respir Care. 2011;56(11):1799-1807.

13. Fabbri LM, Hurd SS. Global strategy for the diagnosis, management and prevention of COPD: 2003 update. Eur Respir J. 2003;22(1):1-2.

14. Nici L, Donner C, Wouters E, et al. American Thoracic Society/ European Respiratory Society statement on pulmonary rehabilitation. Am J Respir Crit Care Med. 2006;173(12):1390-1413.

15. Li F, Harmer P, Fitzgerald K, et al. Tai Chi and postural stability in patients with Parkinson's disease. $N$ Engl J Med. 2012;366(6):511-519.

16. Yeh GY, McCarthy EP, Wayne PM, et al. Tai Chi exercise in patients with chronic heart failure: a randomized clinical trial. Arch Intern Med. 2011;171(8):750-757.

17. Logghe IH, Verhagen AP, Rademaker AC, et al. The effects of Tai Chi on fall prevention, fear of falling and balance in older people: a meta-analysis. Prev Med. 2010;51(3):222-227.

18. Leung RW, McKeough ZJ, Peters MJ, Alison JA. Short-form Sunstyle tai chi as an exercise training modality in people with COPD. Eur Respir J. 2013;41(5):1051-1057.

19. Yan JH, Guo YZ, Yao HM, Pan L. Effects of Tai Chi in patients with chronic obstructive pulmonary disease: preliminary evidence. PLoS One. 2012;8(4):e61806.

20. Chan AW, Lee A, Suen LK, Tam WW. Tai Chi Qigong improves lung functions and activity tolerance in COPD clients: a single blind, randomized controlled trial. Complement Ther Med. 2011;19(1): 3-11.

21. Yao YP. [Effect of Tai Chi Chuan on chronic obstructive pulmonary disease]. Chin J Rehabil Med. 2004;10(7):439-440. Chinese.

22. Zhang LH, Wu JJ, Wang ZC. [Effects of 24-form Tai Chi with respiratory rehabilitation training on chronic obstructive pulmonary disease]. Acta Univ Tradit Med Sin Pharm Shanghai. 2012;2(4):53-56. Chinese.

23. Yeh GY, Roberts DH, Wayne PM, Davis RB, Quilty MT, Phillips RS. Tai chi exercise for patients with chronic obstructive pulmonary disease: a pilot study. Respir Care. 2010;55(11):1475-1482.

24. Maher CG, Sherrington C, Herbert RD, Moseley AM, Elkins M. Reliability of the PEDro scale for rating quality of randomized controlled trials. Phys Ther. 2003;83(8):713-721.

25. Higgins JP, Green S. Cochrane Handbook for Systematic Reviews of Interventions (Version 5.1.0.). Updated March 2011. Available from: http://handbook.cochrane.org/. Accessed January 28, 2014.

26. Higgins JP, Thompson SG, Deeks JJ, Altman DG. Measuring inconsistency in meta-analyses. BMJ. 2003;327(7414):557-560.

27. Chan AW, Lee A, Suen LK, Tam WW. Effectiveness of a Tai Chi Qigong program in promoting health-related quality of life and perceived social support in chronic obstructive pulmonary disease clients. Qual Life Res. 2010;19(5):653-664.

28. Niu R, He R, Luo BL, Hu C. The effect of Tai Chi on chronic obstructive pulmonary disease: a pilot randomized study of lung function, exercise capacity and diaphragm strength. Heart Lung Circ. 2014;23(4):347-352.
29. Ng L, Chiang LK, Tang R, et al. Effectiveness of incorporating Tai Chi in a pulmonary rehabilitation program for chronic obstructive pulmonary disease in primary care: a pilot randomized controlled trial. Eur J Integr Med. 2014;6:248-258.

30. Du ST, Xing B, Wang CX, et al. [The effects of taijiquan practice on the BODE and SGRQ of COPD patients]. Chin J Sports Med. 2013;32(5): 403-407. Chinese.

31. Du ST, Ding LM, Wang CX, et al. [The effects taijiquan practice on exercise endurance and lung function of patients with chronic obstructive pulmonary disease]. Chin J Rehabil Med. 2013;28(4):374 476. Chinese.

32. Li Q, Fang WH, Liu C. [The effect of taijiquan combined with respiratory exercise training on rehabilitation of patients with stable chronic obstructive pulmonary disease]. Chin J Rehabil Med. 2012;27(9): 825-828. Chinese.

33. Gu G, Zhou YM, Wang DL, Chen L, Zhong NS, Ran PX. [Effects of shadow boxing training on exercise endurance and quality of life of patients with chronic obstructive pulmonary disease]. Zhonghua Yi Xue Za Zhi. 2012;92(14):952-955. Chinese.

34. ATS Committee on Proficiency Standards for Clinical Pulmonary Function Laboratories. ATS statement: guidelines for the six-minute walk test. Am J Respir Crit Care Med. 2002;166(1):111-117.

35. Pinto-Plata VM, Cote C, Cabral H, Taylor J, Celli BR. The 6-min walk distance: change over time and value as a predictor of survival in severe COPD. Eur Respir J. 2004;23(1):28-33.

36. Polkey MI, Spruit MA, Edwards LD, et al. Six-minute-walk test in chronic obstructive pulmonary disease: minimal clinically important difference for death or hospitalization. Am J Respir Crit Care Med. 2013;187(4):382-386.

37. Gatchel RJ, Lurie JD, Mayer TG. Minimal clinically important difference. Spine. 2010;35(19):1739-1743.

38. Farrar JT, Portenoy RK, Berlin JA, Kinman JL, Strom BL. Defining the clinically important difference in pain outcome measures. Pain. 2000;88(3):287-294.

39. Copay AG, Subach BR, Glassman SD, Polly DW Jr, Schuler TC. Understanding the minimum clinically important difference: a review of concepts and methods. Spine J. 2007;7(5):541-546.

40. Puhan MA, Chandra D, Mosenifar Z, et al; National Emphysema Treatment Trial (NETT) Research Group. The minimal important difference of exercise tests in severe COPD. Eur Respir J. 2011;37(4):784-790.

41. Holland AE, Hill CJ, Rasekaba T, Lee A, Naughton MT, McDonald CF. Updating the minimal important difference for six-minute walk distance in patients with chronic obstructive pulmonary disease. Arch Phys Med Rehabil. 2010;91(2):221-225.

42. Redelmeier DA, Bayoumi AM, Goldstein RS, Guyatt GH. Interpreting small differences in functional status: the six minute walk test in chronic lung disease patients. Am J Respir Crit Care Med. 1997;155(4): 1278-1282.

43. Mahler DA. How should health-related quality of life be assessed in patients with COPD? Chest. 2000;117(Suppl 2):54S-57S.

44. Ståhl E, Lindberg A, Jansson SA, et al. Health-related quality of life is related to COPD disease severity. Health Qual Life Outcomes. 2005;3:56.

45. Jones PW. St George's Respiratory Questionnaire: MCID. COPD. 2005;2(1):75-79.

46. Schunemann HJ, Puhan M, Goldstein R, Jaeschke R, Guyatt GH. Measurement properties and interpretability of the Chronic Respiratory Disease Questionnaire (CRQ). COPD. 2005;2(1):81-89.

47. Leung RW, Alison JA, McKeough ZJ, et al. A study design to investigate the effect of short-form Sun-style Tai Chi in improving functional exercise capacity, physical performance, balance and health related quality of life in people with chronic obstructive pulmonary disease (COPD). Contemp Clin Trials. 2011;32(2):267-272.

48. Butcher SJ, Jones RL. The impact of exercise training intensity on change in physiological function in patients with chronic obstructive pulmonary disease. Sports Med. 2006;36(4):307-325. 
International Journal of COPD

\section{Publish your work in this journal}

The International Journal of COPD is an international, peer-reviewed journal of therapeutics and pharmacology focusing on concise rapid reporting of clinical studies and reviews in COPD. Special focus is given to the pathophysiological processes underlying the disease, intervention programs, patient focused education, and self management protocols.

This journal is indexed on PubMed Central, MedLine and CAS. The manuscript management system is completely online and includes a very quick and fair peer-review system, which is all easy to use. Visit $\mathrm{http}: / /$ www.dovepress.com/testimonials.php to read real quotes from published authors.

Submit your manuscript here: http://www.dovepress.com/international-journal-of-copd-journal 